\title{
Effect of SDF and SDF/KI Treatment on Microtensile Bond Strength of Bioactive Materials
}

\author{
Tae Kim ${ }^{1}$, Kush Patel ${ }^{1}$ and John C Comisi ${ }^{2 \star}$ \\ 1 The Medical University of South Carolina, James B. Edwards College of Dental Medicine, Charleston, South Carolina, USA \\ 2 Restorative Dentistry, Department of Oral Rehabilitation, The Medical University of South Carolina, James B. Edwards College of Dental Medicine, Charleston, \\ South Carolina, USA
}

*Corresponding author: John C Comisi, Restorative Dentistry, Department of Oral Rehabilitation, The Medical University of South Carolina, James B Edwards College of Dental Medicine, Charleston, South Carolina, USA.

To Cite This Article: Tae Kim, Effect of SDF and SDF/KI Treatment on Microtensile Bond Strength of Bioactive Materials. Am J Biomed Sci \& Res. 2019 - 6(4). AJBSR.MS.ID.001045. DOI: 10.34297/AJBSR.2019.06.001045.

Received: 阱 November 18, 2019; Published: 制 November 27, 2019

\begin{abstract}
Purpose: This pilot study was undertaken to determine whether SDF or SDF/KI treatment of dentin surfaces influenced the microtensile bond strength of commercially available bioactive products.

Methods: The teeth were then randomly divided into three groups of eight depending on the treatment: Advantage Arrest (SDF), Riva Star (SDF/KI), and Control group (Activa materials with and without bonding agent). Samples prepared and microtensile bond strength determined for each sample group.

Results: The results of this study showed a significant statistical difference in microtensile bond strength between the control groups and the Advantage Arrest (SDF) and Riva Star (SDF/KI) treated groups for both restorative materials. Bond strengths further decreased when they applied without the application of a bonding agent.

Conclusion: From the results of this study, the null hypothesis that there would be no significant difference in microtensile bond strength when Activa Bioactive products used with SDF or SDF/KI application, was rejected.
\end{abstract}

Keywords: Silver diamine fluoride; Silver diamine fluoride with Potassium Iodide; Bioactive Materials; Self-etching; Dentin bond strength

\section{Introduction}

Silver Diamine Fluoride (SDF) has been approved in the US for clinical use in recent years, as a desensitizing agent as well as caries arrest [1]. The benefits of SDF are the following:

a) The silver-salts stimulate dentin sclerosis/calcification, and

b) The silver nitrate acts to kill bacteria, and fluoride aids in remineralization and prevention [2].

Recent studies have shown that SDF treatment of dentin reduces the microtensile bond strength to composite but not glass ionomers [3]. There has also been an interest in incorporating potassium iodide (KI) to SDF applications. The reported benefit of using SDF/ KI solution (Riva Star, SDI North America Inc., Itasca, IL) over the SDF only solution (Advantage Arrest, Elevate Oral Care, West Palm
Beach, FL) is that it will not stain the teeth dark after usage while maintaining several antibacterial properties for deeper caries [2]. Bioactive materials have also become quite popular in recent years in the treatment of decay affected teeth. Many clinicians have used the combination of SDF and bioactive materials without full knowledge of the benefit or challenge that this combination may yield. There is a need then to determine if SDF and SDF/KI treated teeth will compromise the adhesion to dentin when bioactive restoratives, like Activa BioActive Restorative, are used.

Activa BioActive Restorative (Pulpdent Corporation, Watertown, MA) is a highly esthetic bioactive hydrophilic composite that bonds chemically to teeth, seals against microleakage, and releases more calcium, phosphate, and fluoride [4]. The company states that Activa is more bioactive than glass ionomers while more durable and fracture resistant than composites. Activa Presto a new bioactive product from Pulpdent that has the properties of 
the Activa Bioactive dual-cure material that is solely light-cured and dispensed from a single syringe. It is reported by the company also to have bioactive properties, that is, calcium, phosphates, and fluoride are released from the hydrophilic resin when in contact with tooth structure and saliva over time. This in-vitro pilot study is to determine whether SDF or SDF/KI treatments influence the microtensile bond strength of Activa BioActive Restorative material (two-paste system in automix syringes) and the new Activa Presto material (single paste syringe dispensing system). The null hypothesis is that there will be no effect on the bond strengths of these materials when applied to SDF or SDI treated teeth.

\section{Materials and Methods}

This study in-vitro study received Institutional Review Board approval (Pro00081912) before the commencement of the study.

\section{Allocation Method}

Twenty-four natural human caries-free extracted molars with fully formed apices, fluorosis, restorations, and fractures were collected and stored in distilled water. Before testing, each tooth was cleaned and stored in Phosphate Buffered Saline (PBS).

\section{Research Protocol}

a) The teeth were then randomly divided into three groups of eight depending on the treatment:

Advantage Arrest (SDF), Riva Star (SDF/KI), and Control group (Activa materials with and without bonding agent). These three groups were then randomly divided into two groups, each depending on the usage of a self-etching bonding agent (Optibond XTR, Kerr Corporation, Orange, CA). The occlusal surfaces of each tooth were reduced until all enamel was removed to reveal the underlying dentin. Then, an automated sandpaper wheel was used to abrade the teeth at 320 Grit and then 600 Grit to expose dentin. Then the tooth was fixed to an acrylic block with Kerr sticky wax. The appropriate materials were applied based on the group the tooth was assigned. The methodology for each group, which followed each company's IFU, is described in Table 1. The restored samples were stored in fresh PBS for seven days.

b) All specimens were then sectioned into individual $1 \mathrm{~mm} \times 1 \mathrm{~mm}$ samples using a sectioning machine with a watercooled diamond disc to create beams. The sample size was ten beams for each group, for a total of 120 beams ( 60 for ActivaRestorative and 60 for Activa-Presto). Each sample was then tested with the microtensile bond strength test device (Bisco Dental Products, Schaumburg, Illinois) to determine the microtensile bond strength. The results were recorded in kgf, with length and width in mm for each sample, and then converted to MPa by using the formula $\mathrm{MPa}=(\mathrm{kgf} \mathrm{x}$ conversion factor 0.0981)/ (length in $\mathrm{cm} \mathrm{x}$ width in $\mathrm{cm}$ ).

\section{Results}

The microtensile bond strength results for Activa-Restorative and Activa-Presto are shown in Table 1 and Table $2 \& 3$ respectively. Data are in units of MPa. The results show that control group with bonding agent yielded highest bond strength at average of $8.98 \mathrm{MPa}$ (Restorative) and 9.91 MPa (Presto), followed by control group without bonding agent at 5.14 MPa (Restorative) and 5.99 MPa (Presto), then SDF with bonding agent at $2.87 \mathrm{MPa}$ (Restorative) and $3.45 \mathrm{MPa}$ (Presto), SDF/KI with bonding agent at $1.65 \mathrm{MPa}$ (Restorative) and $3.10 \mathrm{MPa}$ (Presto), SDF without bonding agent at $0.95 \mathrm{MPa}$ (Restorative) and $1.31 \mathrm{MPa}$ (Presto), and SDI without bonding agent at $0.89 \mathrm{MPa}$ (Restorative) and $1.05 \mathrm{MPa}$ (Presto). During the microtensile bond strength test, all experimental group samples' mode of failure was due to adhesive failure. Only the control group treated with the bonding agent had a few samples with cohesive failure.

Table 1: Methodology of specimen preparation (Four Groups).

a. Every step of the procedure was followed according to the IFU and are as follows:

b. For SDF with bonding agent:

i. After each tooth's occlusal reduction, the tooth was dried with air. Then 1 drop of Advantage Arrest was dispensed into a disposable dish before applying it to the dentin surface. Advantage Arrest was then allowed to soak in for about 1 minute, per manufacturer IFU. The treated surface was then rinsed with water, then dried with air. Following this SDF treatment, Optibond XTR Step 1 Primer was applied to the SDF treated surface for 20 seconds and then air dried. Then Optibond XTR Step 2 Adhesive was applied to the surface for 15 seconds, then air dried, then light cured with 3M Elipar curing light for 10 seconds. Activa BioActive Restorative or Activa Presto depending on the experimental group were then dispensed in a single application to the prepared area with approximately 2 mm of thickness. Restorations were shaped into a rectangular form using a flat glass slide, and then light cured for 20 seconds. The restored tooth was then stored in PBS for 7 days before beam preparation.

c. For SDF without bonding agent:

i. Same as above, but without Optibond XTR application process

d. For SDF/KI group:

Same restoration procedure as above, but without SDF or SDF/KI application and without bonding agent procedure

\begin{tabular}{|c|c|c|c|c|c|c|c|}
\hline Group & Bonding Agent & $\mathbf{N}$ & Mean & Median & Std. Dev & Minimum & Maximum \\
\hline Control & No & 10 & 5.14 & 5.07 & 0.62 & 4.09 & 6.22 \\
\hline
\end{tabular}




\begin{tabular}{|c|c|c|c|c|c|c|c|}
\hline & Yes & 10 & 8.98 & 8.38 & 1.51 & 7.38 & 11.75 \\
\hline SDF & No & 5 & 0.95 & 0.9 & 0.32 & 0.62 & 1.39 \\
\hline & Yes & 10 & 2.87 & 2.23 & 1.26 & 1.84 & 5.48 \\
\hline SDF/KI & No & 5 & 0.89 & 0.92 & 0.24 & 0.49 & 1.13 \\
\hline & Yes & 10 & 1.65 & 1.44 & 0.6 & 1.07 & 2.89 \\
\hline
\end{tabular}

Table 3: Activa-Presto data in MPa.

\begin{tabular}{|c|c|c|c|c|c|c|c|}
\hline Group & Bonding Agent & N & Mean & Median & Std. Dev & Minimum & Maximum \\
\hline Control & No & 10 & 5.99 & 5.73 & 1.02 & 4.69 & 7.98 \\
\hline & Yes & 10 & 9.91 & 9.87 & 1.5 & 7.83 & 12.14 \\
\hline SDF & No & 5 & 1.31 & 1.25 & 0.26 & 1 & 1.58 \\
\hline & Yes & 10 & 3.45 & 2.97 & 1.19 & 2.17 & 5.21 \\
\hline SDF/KI & No & 5 & 1.05 & 1.01 & 0.11 & 0.92 & 1.2 \\
\hline & Yes & 10 & 3.1 & 3.07 & 0.9 & 1.69 & 4.86 \\
\hline
\end{tabular}

Only five samples for the SDF and SDI treated teeth without a bonding agent, could be obtained for testing due to the restorative materials separating from the natural tooth during beam preparation. A two-factor analysis of a variance model (ANOVA) was used to calculate the control and experimental groups, bonding agent, and their interaction within the model. The data was not normally distributed, but all the data is presented normally. The main effects were all significant for both Activa-Restorative and Activa-Presto, as reported in Table 4 \& 5. A Bonferroni adjustment for multiple comparisons was used. Results can be read by comparing the column to the row. For example, the p-value for the Control group without the bonding agent compared to the Control group with the bonding agent is 0.00012 for Activa-Restorative and 0.0003 for Activa-Presto. The only comparisons that were not significant were SDF without bonding agent compared to SDF/KI without bonding agent for both Restorative and Presto and SDF with bonding agent compared to SDF/KI with a bonding agent for Presto.

\begin{tabular}{|c|c|c|c|c|c|c|}
\hline Table 4: Activa- Restorative 2 factor ANOVA test results. \\
\hline $\mathbf{i} / \mathbf{j}$ & Control No & Control Yes & SDF No & SDF Yes & SDI No & SDI Yes \\
\hline Control No & & 0.0012 & $<.0001$ & $<.0001$ & $<.0001$ & $<.0001$ \\
\hline Control Yes & 0.0012 & & $<.0001$ & $<.0001$ & $<.0001$ & $<.0001$ \\
\hline SDF No & $<.0001$ & $<.0001$ & & $<.0001$ & 0.0139 \\
\hline SDF Yes & $<.0001$ & $<.0001$ & $<.0001$ & & $<.0001$ & 0.00019 \\
\hline SDF/KI No & $<.0001$ & $<.0001$ & 1 & $<.0001$ & 0.00051 \\
\hline SDF/KI Yes & $<.0001$ & $<.0001$ & 0.0139 & 0.0019 & 0.0051 & \\
\hline
\end{tabular}

Table 5: Activa-Presto 2 factor ANOVA test results.

\begin{tabular}{|c|c|c|c|c|c|c|}
\hline $\mathbf{i} / \mathbf{j}$ & Control No & SDF No & SDI No & Control Yes & SDF Yes & SDI Yes \\
\hline Control No & & $<.0001$ & $<.0001$ & 0.0003 & $<.0001$ & $<.0001$ \\
\hline SDF No & $<.0001$ & & 1 & $<.0001$ & $<.0001$ & $<.0001$ \\
\hline SDF/KI No & $<.0001$ & 1 & & $<.0001$ & $<.0001$ & $<.0001$ \\
\hline Control Yes & 0.0003 & $<.0001$ & $<.0001$ & & $<.0001$ & $<.0001$ \\
\hline SDF Yes & $<.0001$ & $<.0001$ & $<.0001$ & $<.0001$ & 1 \\
\hline SDF/KI Yes & $<.0001$ & $<.0001$ & $<.0001$ & $<.0001$ & 1 & \\
\hline
\end{tabular}

\section{Discussion}

There has been a surge in the popularity in using Silver Diamine Fluoride (SDF) in the treatment of carious lesions. However, the dark staining of the carious lesion that can occur with its application has led to an interest in reducing this initial discoloration by the application of potassium iodide (KI) to SDF. The popularity of bioactive material use has also been rising. To date, there are no known published works on the bonding of these types of bioactive materials (Activa BioActive or the new Activa Presto) to SDF or SDF/KI. This in-vitro study is to determine whether SDF or SDF/ KI treatments influence the microtensile bond strength of the bioactive products. SDF has been shown to have a significant demineralization and antibacterial effect on these carious lesions [5]. This result is likely due to the metallic silver and silver chloride found in the SDF. It has been demonstrated that the bond 
strengths of Glass Ionomer Cements (GIC) to SDF treated sound or demineralized dentin substrates were not significantly affected when used with SDF [6]. The highest microtensile bond strength in their testing yielded a result of $6.69+2.72 \mathrm{MPa}$ for the group with demineralized dentin with SDF application with light-curing and $6.21+3.28 \mathrm{MPa}$ demineralized dentin without light curing stored for seven days in deionized water. Additional testing of SDF applied to sound dentin without light curing yielded results of $5.19+1.77$ $\mathrm{MPa}$ and $4.53+2.34 \mathrm{MPa}$ with light-curing also stored for seven days in deionized water.

Resin bonding systems have been compared to determine bond strengths when placed after SDF. Total-etch bonding systems, achieve higher bonding strengths to SDF treated teeth than do selfetching bonding agents when restored with composite [2]. One study has demonstrated that there was no significant difference in MSBS between the control untreated specimens and specimens treated with SDF/KI and then restored with resin material with either selfetch or etch and rinse bonding agents [7]. However, this same study found that the etch and rinse bonding agent use did increase the MSBS to the substrates treated with SDF/KI. This result from using an etch and rinse bonding agent likely results in the formation of a continuous, uniform, and thicker hybrid layer as compared to self-etch bonding agents. Another study [8] demonstrated that superficial rewetting with SDF immediately before dentin bonding improved bond strengths. Could this rewetting with SDF before bonding a resin restoration become a needed step in the restorative process when SDF products are placed at an earlier time to attempt to arrest decay? We know that GIC bonds to dentin due to both a chemical and mechanical bond created between hydrogen bonds and various free radicals in collagen and the carboxyl radicals in the GIC [6]. This reapplication of SDF creates a silver and silver oxide interface and that this likely contributes to the improved bond strength to GIC. Can this also apply to resin bonding agents? When SDF is not rinsed after its application to the tooth structure, bond strengths of adhesive to the SDF are severely reduced [8]. The adhesive cannot form stable bonds to dentin, likely due to excessive amounts of SDF present in the dentin. Rinsing of excess SDF, therefore, has been demonstrated to be a crucial step in improving the bonding abilities to dentin adhesives.

This current study demonstrates a notable difference with the usage of SDF or SDF/KI on the bond strength of the bioactive Activa products used when applied with a self-etch bonding agent. Our testing indicates that the use of a bonding agent with the Activa materials is needed, as illustrated in the current instructions for use (IFU) for these products. The maximum results with Activa Restorative and Activa Presto with a self-etch bonding agent to SDF values were $5.48+1.26 \mathrm{MPa}$ and 5.21+1.19 MPa, respectively. The results of Activa Restorative and Activa Presto to SDF/KI, however, were $2.89+.6 \mathrm{MPa}$ and $4.86+.9 \mathrm{MPa}$, respectively. These values illustrate a significantly lower bond strength to SDF/KI with Activa
Restorative material as compared to the Activa Presto material. The addition of KI to the SDF, as illustrated in our testing, affected the bond strengths to both Activa materials. Questions arise from the results of the Selvaraj study [7] as to whether an etch and rinse bonding could have affected the outcomes with these Activa materials since higher bonding strengths have been demonstrated with total-etch bonding agents and traditional resin restorative materials. Self-etch bonding materials may not activate the silver ions effectively to enable more exceptional adhesion. Studies are needed to follow up on this theory.

Additionally, there is no easy way to apply the various bonding tests directly to one another since results in micro-sheer bond strengths (MSBS), may not reflect adequately to the MTBS as reported here. Perhaps a Weibel statistics approach would be a direction to be considered so that the MSBS and MTBS could be compared to identify how these findings could be more clinically relevant. Sirisha, et al. state in that paper that there are a variety of variables that can influence testing outcomes and since no standard format exists for reporting bond strength testing, that there could be many misinterpretations of the data assembled, including the results provided in this study. Further investigations are needed. Within the limits of this study, we have demonstrated that Riva Star (SDF/KI) and Advantage Arrest (SDF) were both statistically significant in reducing the microtensile bond strength of Activa Restorative and Activa Presto materials when applied on dentin. Continued research and replication of this study are necessary with a larger sample size for further validation of the decrease in microtensile bond strength seen in this study. Additional studies comparing the microtensile bond strength of these Activa products using different types of bonding agents, in total-etch, selective etch, and self-etch modes when using SDF or SDKI would complement this study further.

\section{Conclusion}

a. The group with the highest bond strength was the control group with the usage of a bonding agent for both restorative materials. There was a significant statistical difference in microtensile bond strength between the control group with a bonding agent and the control group without a bonding agent for both restorative materials.

b. There were significant statistical differences in microtensile bond strength between the control group and the Advantage Arrest (SDF) and Riva Star (SDKI) treated groups for both restorative materials.

c. Riva Star (SDKI) and Advantage (SDF) Arrest both statistically decreased the microtensile bond strength of ActivaRestorative and Activa-Presto to dentin when applied before restoration. Their bond strengths further decreased when they were applied without the application of a bonding agent. 
d. From the results above, the null hypothesis, of no significant difference in microtensile bond strength when Activa products used with SDF or SDI application, is rejected.

\section{Acknowledgment}

We would like to thank Ms. Abigail Kelly for her help with the statistical analysis.

\section{References}

1. Craig G, Knight G, McIntyre J (2012) Clinical evaluation of diamine silver fluoride/potassium iodide as a dentine desensitizing agent. A pilot study. Australian Dental Journal 57(3): 308-311.

2. Quock R, Barros J, Yang S, Patel S (2012) Effect of Silver Diamine Fluoride on Microtensile Bond Strength to Dentin. Operative Dentistry 37(6): 610-616.

3. Koizumi H, Hamama H, Burrow M (2016) Effect of a silver diamine fluoride and potassium iodide-based desensitizing and cavity cleaning agent on bond strength to dentine. International Journal of Adhesion and Adhesives 68: 54-61.

4. (2019) ACTIVA $^{\mathrm{TM}}$ BioACTIVE-RESTORATIVE ${ }^{\mathrm{TM}}$ Archives - Pulpdent.

5. Yu O, Zhao I, Mei M, Lo E, Chu C (2018) Caries-arresting effects of silver diamine fluoride and sodium fluoride on dentine caries lesions. Journal of Dentistry 78: 65-71.

6. Wang A, Botelho M, Tsoi J, Matinlinna J (2016) Effects of silver diammine fluoride on microtensile bond strength of GIC to dentine. International Journal of Adhesion and Adhesives 70: 196-203.

7. Selvaraj K, Sampath V, Sujatha V, Mahalaxmi S (2016) Evaluation of microshear bond strength and nanoleakage of etch-and-rinse and self-etch adhesives to dentin pretreated with silver diamine fluoride/potassium iodide: An in vitro study. Indian Journal of Dental Research27(4): 421-425.

8. Lutgen P, Chan D, Sadr A (2018) Effects of silver diammine fluoride on bond strength of adhesives to sound dentin. Dental Materials Journal 37(6): 1003-1009. 